Siegfried Wagner-Kompendium 1

herausgegeben von Peter P. Pachl 
Neue Schriftenreihe der

Internationalen Siegfried Wagner Gesellschaft

Band 1 


\title{
Siegfried Wagner-Kompendium 1
}

\author{
Bericht über das erste \\ internationale Symposion \\ SIEGFRIED WAGNER \\ Köln 2001
}

\author{
herausgegeben von \\ Peter P. Pachl
}

Mitarbeit: Lars Kersting

Centaurus Verlag \& Media UG 2003 
Prof. Dr. Peter P. Pachl ist Vizepräsident der Internationalen Siegfried Wagner Gesellschaft e.V. und mit Inszenierungen, Arbeiten für Fernsehen und Rundfunk sowie musikschriftstellerischen Arbeiten befasst. Er ist Lehrbeauftragter an der Musikwissenschaftlichen Fakultät der Universität Bochum und im Fach Theaterwissenschaft an der Universität Bayreuth.

Lars Kersting ist Geschäftsführer und Management-Leiter des künstlerischen Betriebes der Chorakademie am Konzerthaus Dortmund sowie Geschäftsführer der Lobetanz Gesellschaft für Kulturmanagement mbH.

\section{Die Deutsche Bibliothek - CIP-Einheitsaufnahme}

Bibliographische Information der Deutschen Bibliothek:

Die deutsche Bibliothek verzeichnet diese Publikation in der

Deutschen Nationalbibliographie; detaillierte bibliographische Daten

sind im Internet über http://dnb.ddb.de abrufbar

$$
\text { ISBN 978-3-8255-0401-4 ISBN 978-3-86226-465-0 (eBook) }
$$

DOI $10.1007 / 978-3-86226-465-0$

Alle Rechte, insbesondere das Recht der Vervielfältigung und Verbreitung sowie der Übersetzung, vorbehalten. Kein Teil des Werkes darf in irgendeiner Form (durch Fotokopie, Mikrofilm oder ein anderes Verfahren) ohne schriftliche Genehmigung des Verlages reproduziert oder unter Verwendung elektronischer Systeme verarbeitet, vervielfältigt oder verbreitet werden

(C) CENTAURUS Verlags-GmbH. \& Co. KG, Herbolzheim 2003

Abbildungsnachweis im Buch: Die Fotos im Beitrag von Sven Friedrich stammen aus dem Richard-Wagner-Museum mit Nationalarchiv und Forschungsstätte der Richard-Wagner-Stiftung Bayreuth, die Fotos in der Diskussionsrunde "Siegfried Wagner auf dem Theater" aus dem Archiv der ISWG e. V. ("Der Bärenhäuter", "Schwarzschwanenreich", "Wahnopfer" und "Banadietrich" von Sebastian Greuner, "An Allem ist Hütchen Schuld!" von Dietrich Dettmann), alle anderen Fotos aus der der Sammlung Pachl. Wiedergaben von Notenbeispielen aus Klavierauszügen und Partituren erfolgen mit freundlicher Genehmigung des Verlages Max Brockhaus, Bonn-Bad Godesberg.

Umschlag: Bildbearbeitung Achim Bahr

Satz/Layout: Lars Kersting 


\section{Inhalt}

$\begin{array}{ll}\text { Vorwort des Herausgebers } & 7\end{array}$

Fritz Pleitgen: Grußwort des WDR-Intendanten $\quad 9$

Wolfram Steinbeck: Zur Forschung über Siegfried Wagner 11

Eckart Kröplin: Siegfried Wagners Opernwelten in ihrer

Zeitbezogenheit

Peter P. Pachl: Vielfältig mit dem Frühwerk verknüpft. Zur

thematischen Analyse von Siegfried Wagners Opus 15

Bernd Schünemann: Zeitbild im Opernformat

Andrew McCredie: Erkenntnisse zur Frage der Instrumentalmusik in den Opern Siegfried Wagners. Vor-, Zwischenspiele sowie szenographische Musiken

Stephan Hörner: Siegfried Wagners Symphonische Dichtung "Sehnsucht"

Tina Schneeweiß: Tonarten-Symbolik bei Siegfried Wagner, speziell in Opus 15

Christian Dammann: Dichtung und Komposition für eine unausgeglichene Welt - Politische Wirklichkeiten bei Siegfried Wagner

Walter Keller: Baumarten und ihre Funktion in Siegfried Wagners Opern

Isolde Braune: Siegfried Wagners Libretti - Textbücher oder

Dichtungen?

Sabine Busch: Siegfried Wagners eigenwillige Operndramaturgie

Dorothea Renckhoff: ,... und die Seele ging weinend über die

Sümpfe davon." Das Erlebnis Oscar Wilde bei Siegfried Wagner.

Ion Zottos: Clement Harris. Work and Life of Siegfried Wagner's

Friend.

Ralph Philipp Ziegler: Siegfried Wagners „Sonnenflammen“ in

Darmstadt

John Williamson: The Image of Byzantium in „Sonnenflammen “. Ideology in Libretto and Music Reception. 
Sven Friedrich: Siegfried Wagner als Bayreuther Festspielleiter und Regisseur

Leslie Head: Siegfried Wagner-Reception in England. Thoughts and Memories.

Gunther Fleischer: Siegfried Wagners religiöses Weltbild

Roland Dippel: Weltanschauungsreflexe und das Treffen dreier

Religionen in Siegfried Wagners Opus 15

Diskussionsrunden:

I: Werner Andreas Albert, MD Konrad Bach, Hans-Martin Höpner:

Siegfried Wagner im Konzert- und Opernbetrieb

II: Konstanze Lauterbach, Peter P. Pachl:

Siegfried Wagner auf dem Theater

III: Katalin Halmai, Ksenija Lukić, Dagmar Schellenberger, Mechthild Georg, Volker Horn, Thorsten Scharnke, John Wegner, Wolfgang Schmidt: Siegfried Wagner singen

Register 


\section{Vorwort des Herausgebers}

Im letzten Jahrzehnt des 20. Jahrhunderts zeigen sich deutlich verstärkte Tendenzen zu wissenschaftlichen Untersuchungen über das kompositorische und musikdramatische Schaffen von Siegfried Wagner (1869 - 1930).

Die Vorzeichen für ein erstes Symposion über den Komponisten und Dichter, Dirigenten und Regisseur, anlässlich der Uraufführung seiner fünfzehnten Oper Die heilige Linde im Oktober 2001, waren keineswegs rosig. Das wäre denn wohl auch zu verwunderlich gewesen für einen Künstler, der zeit Lebens darunter zu kämpfen hatte, nicht nach seiner eigenen Wert bemessen zu werden, wie es Arnold Schönberg 1912 für Siegfried Wagner treffend formuliert hat, und dessen umfangreiches Oeuvre nach seinem Tode zusehends unter Verschluss gehalten wurde.

Die 1972 - gegen enorme Widerstände der Familie Wagner - gegründete Internationale Siegfried Wagner Gesellschaft e. V. hatte mit Hilfe des WDR Sinfonieorchesters und seines rührigen Managers Hans-Martin Höpner ein umfassendes, einwöchiges wissenschaftliches Symposion geplant und hierfür ohne Schwierigkeiten über vierzig Zusagen namhafter Wissenschaftler erhalten.

Die wohlwollend avisierte Zusage einer Förderung durch die Deutsche Forschungsgemeinschaft erwies sich allerdings als potemkinsches Dorf. Glücklicherweise erklärte sich ein Großteil der Referenten dennoch bereit, auch ohne jegliche Aufwandsentschädigung zu seiner Zusage zu stehen.

Das Ergebnis des einwöchigen Symposions vom 15. bis 20. Oktober 2002 in den Räumen des WDR Köln und der Musikhochschule Köln war verblüffend vielfältig, faszinierend widersprüchlich und spannend bis in die sich anschließenden, häufig auch kontrovers geführten Diskussionen hinein. Bei der Zusammenführung unterschiedlicher Forschungsansätze in einer komplexen Untersuchung des Sohnes von Richard Wagner und Enkels von Franz Liszt unter musikologischen, literaturund theaterwissenschaftlichen, historischen und soziologischen Gesichtspunkten, erwies sich Siegfried Wagner und sein Werk in der Tat als ein noch lange nicht ausgeschöpfter Forschungsgegenstand, mit allerlei Querverweisen auf künstlerische und politische Entwicklungen des späten 19. und des ersten Drittels des 20. Jahrhunderts.

Über 30 Experten aus verschiedenen Disziplinen der Geisteswissenschaft und der Musik(theater)-Praxis referierten und diskutierten über Aspekte des Siegfried Wagnerschen Oeuvres. Hierzu zählten die Musikologen Prof. Dr. Andrew McCredie (München), Prof. Dr. Ion Zottos (Athen), Dr. Walter Keller (Birmensdorf), Prof. Dr. Eckart Kröplin (Dresden), Tina Schneeweiß (München), Dr. Sven Friedrich (Bayreuth), Dr. Ralph Philipp Ziegler (Bad Orb), Prof. Dr. Wolfram Steinbeck (Bonn), Dr. Stefan Hörner (München), Prof. Dr. John Williamson (Liverpool), Prof. Dr. Peter P. Pachl (Berlin), der Jurist und Musikschriftsteller 
Prof. Dr. Bernd Schünemann (Eching a. A.), die Dirigenten Prof. Werner Andreas Albert (Brisbane), Leslie Head (Brighton), MD Konrad Bach (Gossel), die Sängerdarsteller Prof. Mechthild Georg (Köln), Katalin Halmai (Budapest), Ksenija Lukić (Berlin), Dagmar Schellenberger (Berlin), John Wegner (Düsseldorf), Thorsten Scharnke (Münster), Volker Horn (Berlin), Ks. Wolfgang Schmidt (Düsseldorf), die Regisseurin Konstanze Lauterbach (Berlin), der Kulturmanager Hans-Martin Höpner (Köln), die Dramaturgen Dorothea Renckhoff (Köln), Dr. Sabine Busch (München), Roland Dippel (München), die Literaturwissenschaftlerin Mag. Isolde Braune (Eldingen) und der Theologe Dr. Gunther Fleischer (Bonn).

Die Drucklegung dieses Symposions wissenschaftlicher Koryphäen zum Oeuvre des Bayreuther Komponisten als Siegfried Wagner Kompendium I erfolgt in der Reihenfolge ihres Auftretens in Köln. Die sich an die Referate anschließenden Diskussionen sind verkürzt wiedergegeben. Nur die Gesprächsrunden wurden komplett an das Ende der Referate gerückt. Das Siegfried Wagner Kompendium I bildet den Auftakt zu einer neuen Schriftenreihe der Internationalen Siegfried Wagner Gesellschaft e. V.

\section{Herzlicher Dank gebührt den Sponsoren, die diese Drucklegung ermöglicht haben:}

Der Friedrich Bauer Beteiligungs-GmbH, Himmelkron und hier insbesondere Herrn Regierungspräsidenten a. D. Wolfgang Winkler, der Oberfranken-Stiftung, und hier insbesondere Herrn Regierungspräsidenten Hans Angerer, der Firma RAPS GmbH und Co KG, Kulmbach, und hier insbesondere Herrn Horst Kühne, sowie einer Reihe von privaten Spendern, darunter die ISWG-Mitglieder Sandra Ehrens, Friedrich Flessa, Dr. Hellmut Karge, Evelyne Lubert, Klaus-Ulrich Mück, Prof. Werner Müller, sowie die Referenten Dr. Walter Keller und Konstanze Lauterbach.

Großer Dank gebührt den Referenten, die ihre Leistungen ohne Aufwandsentschädigung erbracht haben.

Herrn Prof. Dr. Bernd Schünemann gehört der Dank für die Vermittlung des Projektes an den Centaurus-Verlag.

Sehr herzlich danke ich meinem Mitherausgeber, Herrn Lars Kersting und seinen Helferinnen, vor allem Magdalena Konkel und Silvia Engels, für die schriftliche Erfassung der Diskussionen und die Korrekturen. Herrn Achim Bahr gebührt Dank für das Lektorieren, für seine diversen Anregungen und seine Ergänzung des Kompendiums um die Chronologie, sowie Rebecca Broberg für das Lektorieren der englischen Seiten.

Last but not least danke ich der Verlegerin, Frau Britta Schulz, für Ihr persönliches Engagement am Zustandekommen dieses Buches. 